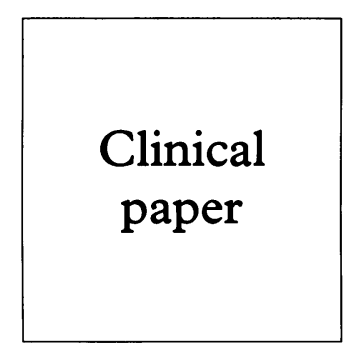

\title{
General practitioners' immediate management of men presenting with urethral symptoms
}

\author{
J G Ainsworth, T Weaver, S Murphy, A Renton
}

Objectives: To describe the immediate reported management, by general practitioners (GPs), of men presenting with symptoms of urethral discharge, or dysuria only.

Subjects: All 692 GPs in practice in Brent, Harrow, Ealing, Hammersmith, and Hounslow (UK).

Method: Data were collected using a GP completed questionnaire concerning the management of the last male patient seen, aged less than 40 years, complaining of urethral discharge, and the last male patient under 40 years complaining of dysuria only.

Results: The response rate among GPs was $52 \%$. Fifty three per cent of men with urethral symptoms, $86 \%$ of men with a urethral discharge and $24 \%$ with dysuria only, were identified by GPs and referred without investigation or treatment to a genitourinary medicine clinic. Of men with dysuria only, $93 \%$ of investigations by GPs were reported to include a mid-stream urine (MSU) specimen for bacteriology, and $19 \%$ a urethral swab for chlamydia. Seventy eight per cent of GPs reported using treatments with a broad spectrum antibiotic, $53 \%$ with trimethoprim, whilst $14 \%$ of GPs reported using a tetracycline in common use to treat non-gonococcal urethritis. Urine specimens were reported to be "culture positive" in $41 \%$ of men who had an MSU specimen tested, and $15 \%$ of men who had a urethral swab tested were reported to be chlamydia positive.

Conclusion: The GPs included in this study were not a full sample, or representative of all the GPs, and the data are retrospective. Nevertheless, we found a large difference in GPs reported management for men with urethral symptoms according to whether or not urethral discharge was a reported complaint. Reported management is likely to be, at least, indicative of actual management. Therefore, the results suggest that assessment by GPs of men presenting with dysuria should be explored and more appropriate management strategies defined. (Genitourin Med 1996;72:427-430)

Keywords: general practitioner; urethra; symptoms

Sexually Transmitted Disease Research Group J G Ainsworth

Academic Dept of Genitourinary Medicine, St Mary's Hospital Medical School, Paddington, London W2, UK TWeaver A Renton

Dept of Genitourinary Medicine, Central Middlesex Hospital, Park Royal, London NW10, UK S Murphy Accepted for publication 4 September 1996

\section{Introduction}

HIV and sexual health is a key priority area within the UK government's strategy: The Health of the Nation, ${ }^{1}$ and the recent report, Making London Better, ${ }^{2}$ identified the need to expand the role of primary health care in the capital.

In our experience a substantial proportion of men with sexually transmitted infections present first to their general practitioner (GP). We have also noted that GPs can misdiagnose urethritis in men as urinary tract infection (UTI). Urethral discharge and dysuria are the commonest symptoms of sexually transmitted urethritis in men. Whereas discharge is highly associated with urethritis, dysuria is experienced commonly with either urethritis or a UTI.

We therefore conducted a survey to determine the immediate management by GPs of men who present to them complaining of urethral discharge, or dysuria. We wished to determine the proportions of patients with the most common urethral symptoms who are referred to a genitourinary medicine (GUM) clinic at the initial consultation, and the extent to which the diagnosis suspected by the GPs on the basis of symptom pattern influenced their immediate referral practice, choice of investigation, and antibiotic treatment.

\section{Method}

All GPs practising in Brent and Harrow health agency and Ealing, Hammersmith and Hounslow health agency were sent a questionnaire by mail. A total of $692 \mathrm{GPs}$ were identified representing all GPs working in 336 practices. They were asked to indicate their immediate management, at the first consultation, of two groups of patients: the last male patient, less than 40 years of age, whom they had seen complaining of symptoms of "dysuria only", and the last male patient, under 40 years, whom they had seen complaining of a "urethral discharge". General practitioners identified the initial investigations they had undertaken, and each result as positive or negative, and the antibiotic treatments they had prescribed according to choices in the questionnaire. Men under 40 were selected so that the management by GPs of a population comparable in age to direct GUM clinic attenders could be studied. In addition, GPs were asked to report the age of the last male patient they had seen in each of the patient groups, and estimate the number of men they would see in an average six month period in each of the groups. GPs were also asked to indicate their usual practice concerning partner notification both when consulted by a man with dysuria in whom they had diagnosed urethritis, and a 
man with urethral discharge. We intended the designation "dysuria only" to refer to a man who had complained of symptoms of dysuria without urethral discharge, but who might complain of unspecified symptoms such as urethral irritation, or frequency.

\section{Analysis}

Questionnaires were coded, entered in to a computer, and analysed using SAS software. Statistical tests for differences in proportions used chi square test, or Fisher's exact test. The Kruskal Wallis chi square test was used for differences in the central tendency of distributions of continuous variables.

\section{Results}

Response of GPs

Completed questionnaires were received from 358 GPs in 208 practices giving an overall response rate of $52 \%$ for GPs and $61 \%$ for practices. The distribution of the GPs across location, size of practice from which the GP was operating, gender of the GP, and whether or not the practice was fundholding is shown in table 1. Female GPs were more likely to respond, as were GPs from practices where four or more GPs were working.

\section{Numbers of men seen in each study group}

The distribution of the numbers of men in the dysuria only and discharge groups that GPs reported having seen in the six month period is shown in table 2. The median number of men less than 40 years old with dysuria only seen by 343 GPs who provided an estimate was two (0-30). The median number of men of similar age who had a discharge and were seen by the 336 GPs who provided an estimate was one (0-12).

Age of last men seen in each study group Two hundred and twenty-eight GPs reported that the age of the last man they saw with dysuria only. The median reported age was 32 years (range: 15-40). One hundred and ninety six GPs reported the age of the last man they saw with a urethral discharge. The median reported age was 28 years (range: 16-40). Men with dysuria only were reported as significantly older than those with urethral discharge $(p<$ $0 \cdot 0001$, Kruskal-Wallis chi square $57 \cdot 48$, df 1 ).

Table 1 Characteristics of study sample and study respondents

\begin{tabular}{lllc}
\hline & & $\begin{array}{l}\text { Number } \\
\text { GPs contacted }\end{array}$ & $\begin{array}{l}\text { Respondents } \\
n(\%)\end{array}$ \\
\hline Location & Brent & 179 & $95(53)$ \\
& Ealing, & & \\
& Hammersmith, & & \\
& Hounslow & 409 & $208(51)$ \\
Practice size & Harrow & 104 & $55(53)$ \\
& 1 GP & 168 & $74(44)$ \\
GP gendert & 4+ GPs & 318 & $152(48)$ \\
Fundholder & Male & 206 & $132(64)$ \\
& Female & 432 & $210(49)$ \\
& Yes & 260 & $148(57)$ \\
& No & 81 & $47(58)$ \\
\hline
\end{tabular}

${ }^{\star}$ Chi square (heterogeneity) $=17 \cdot 2,1 \mathrm{df}: \mathrm{p}=0.00003$ †Chi square (heterogeneity) $=4 \cdot 16,1 \mathrm{df}: \mathrm{p}=0.04$.
Table 2 Estimated frequency of presentation of men under forty from each study group in an average six month period

\begin{tabular}{|c|c|c|}
\hline & Dysuria only & Discharge \\
\hline $\begin{array}{l}\text { Men seen in } \\
6 \text { months }\end{array}$ & $\begin{array}{l}\text { No GPs } \\
\text { reporting } \\
n=343 \\
n(\%)\end{array}$ & $\begin{array}{l}\text { No GPs } \\
\text { reporting } \\
n=336 \\
n(\%)\end{array}$ \\
\hline $\begin{array}{r}0 \\
1 \\
2 \\
3 \\
4 \\
5 \\
6 \\
7 \\
8 \\
9 \\
10 \\
12 \\
15 \\
20 \\
21 \\
30\end{array}$ & $\begin{array}{c}52(15) \\
67(20) \\
76(22) \\
50(15) \\
27(8) \\
16(5) \\
24(7) \\
0 \\
2 \\
1(5) \\
16(5) \\
4 \\
1 \\
4 \\
1 \\
2\end{array}$ & $\begin{array}{r}79(24) \\
113(34) \\
66(20) \\
34(10) \\
21(6) \\
6(2) \\
9(3) \\
1 \\
0 \\
3 \\
3 \\
1\end{array}$ \\
\hline
\end{tabular}

Reported immediate management of the last patient seen in each study group

Dysuria As shown in table 3, overall $53 \%$ of GPs referred men from the two study groups, without investigation or treatment, to a GUM clinic, $24 \%$ referring men with dysuria only, and $86 \%$ referring men with an urethral discharge.

One hundred and ninety six (72\%) GPs reported that their usual management of men with dysuria did not include immediate referral Management comprised investigation only, by $79(29 \%)$ of GPs, treatment only, by $19(7 \%)$, and treatment with investigation by $48(36 \%)$ of GPs. Thus, immediate referral with investigation, or treatment was reported by only 11 (4\%) of GPs, and immediate referral with investigation and treatment by two $(1 \%)$ of GPs.

Urethral discharge Thirty (12\%) GPs reported that their management of men with urethral discharge did not include immediate referral. Management comprised investigation only, by nine $(4 \%)$ of GPs, treatment only, by eight $(3 \%)$, and investigation and treatment by 13 (5\%) of GPs. Thus, immediate referral with investigation, or treatment was reported by only four $(1 \cdot 6 \%)$, and immediate referral with investigation and treatment was not reported by any GPs.

Investigation and treatment of men with dysuria All the 188 GPs who reported carrying out immediate investigation of men with dysuria, specified investigations undertaken. In addi-

Table 3 Reported immediate management of last man aged under 40 years complaining of dysuria only and last man under 40 complaining of urethral discharge

\begin{tabular}{|c|c|c|}
\hline & Dysuria only & Discharge \\
\hline Management modality & $\begin{array}{l}\text { No GPs } \\
n=274 \\
n(\%)\end{array}$ & $\begin{array}{l}\text { No GPs } \\
n=241 \\
n(\%)\end{array}$ \\
\hline $\begin{array}{l}\text { Referral only } \\
\text { Treatment only } \\
\text { Investigation only } \\
\text { Referral }+ \text { treatment } \\
\text { Referral }+ \text { investigation } \\
\text { Treatment }+ \text { investigation } \\
\text { All three }\end{array}$ & $\begin{array}{l}65(24) \\
19(7) \\
79(29) \\
2 \\
9(2) \\
98(36) \\
2\end{array}$ & $\begin{array}{c}207(86) \\
8(3) \\
9(4) \\
3(1) \\
1 \\
13(5) \\
0\end{array}$ \\
\hline
\end{tabular}


tion, five GPs reported information on investigations, without having indicated that investigation was part of their management. Fourteen (7\%) carried out urinalysis alone, and 48 (25\%) sent an MSU for bacteriology as the only investigation. Ninety five (49\%) carried out urinalysis and sent an MSU, and eight ( $4 \cdot 1 \%)$ sent both an MSU and a urethral swab for chlamydia. Twenty eight $(14 \cdot 5 \%)$ carried out all three investigations. Thus, $93 \%$ of all investigation regimes included an MSU, and $19 \%$ a urethral swab for chlamydia.

Results of urinalysis were reported by 109 GPs, $61(56 \%)$ as showing proteinuria/microscopic haematuria. Results of MSU culture were reported in 138 cases, $57(41 \%)$ as culture positive. Results of swabs for chlamydia were reported by 27 GPs four (15\%) as positive.

We were surprised at the high proportion of reported positive MSU cultures, and were interested to know whether the frequency of reported infection increased with age as has been previously described. ${ }^{3}$ GPs reported the ages of the men in whom urine culture was positive to be older (median 35 years, range 16-40) than men in whom urine culture was negative (median 32 years, range 20-40). This difference was significant $(p<0.05$ Kruskal-Wallis, chi square $=6.06,1 \mathrm{df}$ ). In addition, men for whom an MSU was sent were older (median 35 years, range 16-40) than men for whom an MSU was not sent (median 30 years, range 19-40). This difference was significant ( $\mathrm{p}<0.0001$ Kruskal-Wallis, chi squared = $22 \cdot 9,1 \mathrm{df}$ ).

Of the 121 GPs who reported undertaking immediate treatment of their last male patient under 40 men complaining of dysuria information on the antibiotic used was provided by 118. Sixty-two (53\%) used trimethoprim, 16 $(14 \%)$ used a tetracycline, $14(12 \%)$ used ciprofloxacin, $11(9 \%)$ used a cephalosporin, five (4\%) used amoxycillin, and five (4\%) used another antibiotic.

\section{Investigation and treatment of men with urethral discharge}

Of the 23 GPs who reported undertaking immediate investigation of their last male patient aged under 40 years complaining of urethral discharge, all provided information on the type of investigation performed. Twenty one (91\%) sent a swab for Chlamydia trachomatis and $23(100 \%)$ a swab for gonorrhoea.

Results of gonorrhoea culture were reported by 22 GPs, one as positive. Results of chlamydia swab were reported by 20 GPs, three as positive.

Of the 24 GPs who reported providing immediate treatment for men with urethral discharge, nine reported using a tetracycline, seven ciprofloxacin, seven amoxycillin, and one each erythromycin and a cephalosporin. One GP reported using an antibiotic recorded as "other."

\section{Management of partners}

GPs were asked to indicate their usual practice with regard to sexual partners when consulted by men with dysuria in whom they had diag-
Table 4 Advice on action by female partners

\begin{tabular}{|c|c|c|}
\hline & Dysuria & Discharge \\
\hline Management modality & $\begin{array}{l}\text { No GPs } \\
\text { reporting } \\
(n=311) \\
n(\%)\end{array}$ & $\begin{array}{l}\text { No } G P s \\
\text { reporting } \\
(n=298) \\
n(\%)\end{array}$ \\
\hline $\begin{array}{l}\text { Advise consult GP only } \\
\text { Advise consult GUM only } \\
\text { Advise consult both } \\
\text { neither }\end{array}$ & $\begin{array}{r}149(48) \\
89(29) \\
36(12) \\
37(12)\end{array}$ & $\begin{array}{c}108(36) \\
141(48) \\
13(4) \\
36(12)\end{array}$ \\
\hline
\end{tabular}

nosed urethritis, and by men with urethral discharge. As shown in table $4,88 \%$ of GPs would advise some action to such men with dysuria, or men with urethral discharge, either that their sexual partner should attend a GUM clinic, or their GP, or both, but the majority of GPs would not solely advise that partners should attend a GUM clinic.

\section{Discussion}

Two shortcomings of our study need to be acknowledged at the outset. Firstly, the response rate achieved was $52 \%$, while we believe this is respectable for a complete sample of GPs in such a large area, those GPs included in the study clearly do not represent a random sample, and there is evidence of overrepresentation of GPs working in group practices and female GPs. Whilst our findings cannot thus be taken as representative overall they do describe reported practice of more than half of all the GPs. Secondly, the data are retrospective and based on estimates and memory. It has not been possible to validate the data and indeed it is unlikely that additional examination of the records would help. Nonetheless, we feel that the reported management is likely to be, at least, indicative of the actual management strategies used by the GPs.

When considering this study we noted that dysuria is a common symptom both in men with urinary tract infection (UTI) and in men with urethritis presenting to GPs, although urethritis may be characterised by urethral discharge. At an initial consultation GPs may not be able to distinguish between urethritis and UTI based on history alone, but the presence of meatal inflammation, or urinary threads provides evidence of urethritis. ${ }^{4}$ Furthermore, a sexual history may provide important circumstantial information to help diagnosis. In the current study, we did not ask whether GPs had taken a sexual history, or had examined their patients, or their urine. Whether information, thus obtained, had influenced GPs to identify urethritis in a proportion of men with dysuria is unclear.

We found that $86 \%$ of GPs reported immediate referral of their last male patient aged under 40 years complaining of urethral discharge. This suggests that GPs may, in general, immediately refer men whom they consider to have urethritis. In contrast, it may be that GPs do not consider urethritis the most likely diagnosis in men with dysuria whom they choose to investigate and/or treat. 
Seventy two per cent of GPs reported management for men with dysuria that did not include immediate referral. Empirical treatment combined with investigation was the commonest practice. It was also rare for GPs to report referral at the same time as initiating investigation, and, or treatment (13 (6\%) of GPs reported this practice). Therefore, where GPs reported investigations and treatments these were usually carried out for men with dysuria who were not referred. Ninety three per cent of such investigations included an MSU, and $19 \%$ a urethral swab for chlamydia. In $78 \%$ of cases where GPs treated patients immediately, treatment was with a broad spectrum antibiotic (53\% prescribing trimethoprim), while only $14 \%$ prescribed a tetracycline. These findings are consistent with the view that the majority of GPs formed an initial diagnosis of urinary tract infection in men under 40 years presenting with dysuria only, although $59 \%$ of the GPs reporting investigation by MSU in these men, reported negative results.

Moreover, the majority of GPs reported that it was not their usual practice to advise men with urethral symptoms, in whom they had diagnosed urethritis, that their female partners should attend a GUM clinic, although this may indicate that GPs usually refer men, in whom they have made a presumptive diagnosis, and assume appropriate contact tracing is made by GUM physicians.

We suggest that urethritis should be considered more often as a possible diagnosis in this population of men and that GPs might consider a lower threshold for immediate GUM clinic referral. An underlying urethritis can be immediately assessed in the GUM setting, by Gram stain of a urethral smear, and by the urine two-glass test. ${ }^{4}$ These findings justify further assessment of GPs management of men presenting with dysuria in order to clarify the optimum management practice.

We thank Alan Giles for clerical assistance.

1 Secretary of State for Health. The Health of the Nation. A Strategy for Health in England. London, 1992, HMSO. 2 Sir Bernard Tomlinson. Report of the enquiry into London's HMSO.

3 Freedman LR, Phair JP, Hamilton HB, Nefzger MD. The epidemiology of urinary tract infections in Hiroshima. Yale $\mathcal{F}$ Biol Med 1965;37:262-82.

4 Munday PE, Altman DG, Taylor-Robinson. Urinary abnormalities in non gonococcal urethritis. Brf Venereal Dis 1981;6:387-90. 